\title{
Residue Integrals and Waring's Formulas for Algebraic or Even Transcendental Systems
}

\author{
A. A. Kytmanov ${ }^{* 1}$, A. M. Kytmanov ${ }^{\dagger 2}$, and E. K. Myshkina ${ }^{\ddagger 2}$ \\ ${ }^{1}$ Institute of Space and Information Technology, Siberian Federal University, \\ 79 Svobodny av., 660041 Krasnoyarsk, Russia \\ ${ }^{2}$ Institute of Mathematics and Computer Science, Siberian Federal University, \\ 79 Svobodny av., 660041 Krasnoyarsk, Russia
}

\begin{abstract}
The present article is focused on the study of a special class of systems of nonlinear transcendental equations for which classical algebraic and symbolic methods are inapplicable. For the purpose of study of such systems we develop a method for computing residue integrals with integration over certain cycles. We describe conditions under which the mentioned residue integrals coincide with power sums of the inverses to the roots of a system of equations (i.e., multidimensional Waring's formulas). Based on this we develop an algorithm that computes such power sums without computing the roots. As an application of the suggested method, we consider a problem of finding sums of multi-variable number series.
\end{abstract}

2010 MSC: 65H10, 68W30

Keywords: systems of transcendental equations; residue integrals; power sums; Waring's formulas

\section{Introduction}

The problem of elimination of unknowns from systems of nonlinear algebraic equations is a classical algebraic problem. Its solution based on the notion of resultant was developed in works of Silvester and Bézout. This method was described in detail in the classical Van der Waerden's monograph [25]. In the middle of the 20th century, B. Buchberger suggested a new elimination method based on the notion of a Gröbner basis. Nowadays it is one of the main elimination methods in polynomial computer algebra (see, e.g., $[1,4]$ ).

In the 1970s in [2] L. A. Aizenberg proposed a new elimination method based on the multidimensional residue theory, namely on the formulas of multidimensional logarithmic residue and Grothendieck residue. The basic idea of the method was to find certain residue integrals

\footnotetext{
*E-mail: aakytm@gmail.com

$\dagger$ E-mail: akytmanov@sfu-kras.ru

$\ddagger$ E-mail: elfifenok@mail.ru
} 
connected to the power sums of roots of a given system of equations without finding the roots themselves. (The formulas for computing power sums may vary depending on the given system of equations.) Then, using the classical recurrent Newton formulas, one can construct a polynomial whose roots coincide with the first coordinates of the roots of the given system with the same multiplicity (i.e., resultant). This method does not increase the multiplicity of roots in comparison with the classical method (see, e.g., [25]). Its further developments were implemented in [3], [24], and [5].

In applied problems of chemical kinetics systems of transcendental equations, namely, systems consisting of exponential polynomials [6,8] (Zeldovich-Semenov model, etc.) arise as well. However, the elimination method developed in $[3,5,24]$ cannot be applied to this kind of systems. One of the obstacles is the fact that the set of roots of a system of $n$ transcendental equations in $n$ variables is, in general, infinite. Moreover, multi-Newton sums (with powers in $\mathbb{N}^{n}$ ) of the roots of such systems lead usually to divergent series. At the same time, the multidimensional logarithmic residue and Grothendieck residue formulas are not applicable to the residue integrals that arise in such kind of systems which means that one is unable to calculate power sums of the roots. Therefore, the known methods have to be modified significantly in order to be applied to such systems. In particular, one has to be able to compute power sums of the inverses to the roots (without finding the roots themselves). Then, using the obtained formulas together with analogs of recurrent Newton formulas and Waring's formulas for entire functions of a complex variable (see [5, Ch. 1]), one can construct resultant, which is also an entire function. But nevertheless, the formulas for finding power sums are still the main component of the method.

Classical Waring's formulas express power sums of the roots of a polynomial in terms of its coefficients (see, e.g., [25]). Multidimensional analogs of Waring's formulas for certain types of algebraic systems were developed in [10].

In the works $[7,11,13-18]$ simple enough classes of systems of equations containing entire or meromorphic functions were considered. An algorithm that computes the residue integrals and applies to them the recurrent Newton formulas was given in [12]. In [9] the developed methods were applied to study of a system (consisting of exponential polynomials) that arises in Zeldovich-Semenov model.

We now present a more precise review of the known results. In [18] the authors considered the system of functions:

$$
f_{1}(z), \ldots, f_{n}(z)
$$

where $z=\left(z_{1}, \ldots, z_{n}\right)$. Each $f_{j}(z)$ is analytic in the neighborhood of $0 \in \mathbb{C}^{n}$ and is defined by

$$
f_{j}(z)=z^{\beta^{j}}+Q_{j}(z), \quad j=1, \ldots, n,
$$

where $\beta^{j}=\left(\beta_{1}^{j}, \ldots, \beta_{n}^{j}\right)$ is a multi-index with integer nonnegative coordinates, $z^{\beta^{j}}=z_{1}^{\beta_{1}^{j}} \ldots \cdot z_{n}^{\beta_{n}^{j}}$, and $\left\|\beta^{j}\right\|=\beta_{1}^{j}+\ldots+\beta_{n}^{j}=k_{j}, j=1, \ldots, n$. Functions $Q_{j}$ are expanded in a neighborhood of origin into an absolutely and uniformly converging Taylor series of the form

$$
Q_{j}(z)=\sum_{\|\alpha\|>k_{j}} a_{\alpha}^{j} z^{\alpha}
$$

where $\alpha=\left(\alpha_{1}, \ldots, \alpha_{n}\right), \alpha_{j} \geqslant 0, \alpha_{j} \in \mathbb{Z}$, and $z^{\alpha}=z_{1}^{\alpha_{1}} \cdot \ldots \cdot z_{n}^{\alpha_{n}}$.

The formulas for computing residue integrals

$$
J_{\beta}=\frac{1}{(2 \pi i)^{n}} \int_{\gamma(r)} \frac{1}{z^{\beta+U}} \cdot \frac{d f}{f}
$$


(here $i=\sqrt{-1}$ ) in terms of the coefficients of $Q_{j}(z)$ were obtained. Here $\gamma(r)=\{z=$ $\left.\left(z_{1}, \ldots, z_{n}\right):\left|z_{j}\right|=r_{j}, j=1, \ldots, n\right\}$ and $U=(1, \ldots, 1)$ is the unit vector.

Multidimensional Newton formulas for such systems were obtained in [11] and [12].

A class of systems containing the functions

$$
f_{j}(z)=\left(z^{\beta^{j}}+Q_{j}(z)\right) e^{P_{j}}, \quad j=1, \ldots, n .
$$

was considered in [15] and [13]. A method for finding residue integrals for such systems was given in [13].

In the present work we compute residue integrals for a specific kind of systems of $n$ transcendental equations, and deduce from this computation (provided such series converge) the values of the sums of series (with powers in $(-\mathbb{N})^{n}$ ) consisting of the roots of such systems which do not belong to coordinate subspaces. In other words, we generalize the statements from $[7,11,13,15,18]$ to a wider class of systems of transcendental equations, where instead of the monomials $z^{\beta^{j}}$ in (1) we consider products of linear functions. Our objectives are to obtain formulas for computing residue integrals, to study the connection between residue integrals and power sums of the inverses to the roots (Waring's formulas), and to introduce a scheme for elimination of unknowns from the considered class of systems.

\section{Calculation of residue integrals}

In this section, we introduce the class of systems of transcendental equations that will be considered in this work. A. Tsikh considered its algebraic analog in [23] (see also [5, Theorems 8.5, 8.6]) and studied the number of its common roots in $\left(\mathbb{P}^{1}(\mathbb{C})\right)^{n}$. Theorem 2 shows that for any such system the residue integral $J_{\gamma}(t)$ (where $t>0$ is sufficiently small) can be computed by means of converging series of Taylor coefficients of the functions contained in the initial system.

For $z=\left(z_{1}, \ldots, z_{n}\right) \in \mathbb{C}^{n}$ consider a system of functions

$$
\left\{\begin{array}{l}
f_{1}(z)=q_{1}(z)+Q_{1}(z) \\
\cdots \\
f_{n}(z)=q_{n}(z)+Q_{n}(z)
\end{array}\right.
$$

where $Q_{j}(z)$ are entire functions and

$$
q_{j}\left(z_{1}, \ldots, z_{n}\right)=\left(1-a_{j 1} z_{1}\right)^{m_{j 1}} \cdot \ldots \cdot\left(1-a_{j n} z_{n}\right)^{m_{j n}}
$$

for $j=1, \ldots, n$. Here $m_{j k}$ are positive integers and $a_{j k}$ are complex numbers, such that $a_{j k} \neq a_{s k}, j \neq k$.

Let $J=\left(j_{1}, \ldots, j_{n}\right)$ be a multi-index where $\left(j_{1} \ldots j_{n}\right)$ is a permutation of $(1 \ldots n)$. Then by $a_{J}$ we denote the vector $\left(a_{1 j_{1}}, \ldots, a_{n j_{n}}\right)$. For each $i$ we define a function

$$
h_{j}(z)= \begin{cases}q_{j}(z), & \text { if } a_{j k} \neq 0 \text { for each } k \\ q_{j}(z) \cdot \frac{1}{z_{k_{1}}} \cdot \ldots \cdot \frac{1}{z_{k_{s}}}, & \text { if } a_{j k_{1}}=\ldots=a_{j k_{s}}=0 .\end{cases}
$$

The system

$$
h_{i}(z)=0, \quad i=1, \ldots, n
$$


has $n$ ! isolated roots (computed without multiplicities) in $\left(\mathbb{P}^{1}(\mathbb{C})\right)^{n}$. Since $\mathbb{P}^{1}(\mathbb{C})$ is a compactification of the complex plane $\mathbb{C}$, then $\left(\mathbb{P}^{1}(\mathbb{C})\right)^{n}$ is one of the known compactifications of $\mathbb{C}^{n}$. The roots of (4) are

$$
\tilde{a}_{J}= \begin{cases}\left(1 / a_{1 j_{1}}, \ldots, 1 / a_{n j_{n}}\right), & \text { if } a_{k j_{k}} \neq 0 \text { for each } k=1, \ldots, n, \\ \left(1 / a_{1 j_{1}}, \ldots, \infty_{\left[i_{1}\right]}, \ldots, \infty_{\left[i_{k}\right]}, \ldots, 1 / a_{n j_{n}}\right), & \text { if } a_{i_{1} j_{i_{1}}}=\ldots=a_{i_{k} j_{i_{k}}}=0,\end{cases}
$$

where $k, j=1, \ldots, n$, and $J=\left(j_{1}, \ldots, j_{n}\right)$. Note that we write $\infty$ (as a point in $\left.\mathbb{P}^{1}(\mathbb{C})\right)$ in $\tilde{a}_{J}$ whenever $a_{k j_{k}}=0$.

By $\Gamma_{h}$ we denote the cycle

$$
\Gamma_{h}=\left\{z \in \mathbb{C}^{n}:\left|h_{j}(z)\right|=r_{j}, r_{j}>0, j=1, \ldots, n\right\} .
$$

Now we define the cycle $\Gamma_{h, \tilde{a}_{J}}$ by

$$
\left\{\begin{array} { l } 
{ | l _ { 1 } | = r _ { 1 } , } \\
{ \cdots } \\
{ | l _ { n } | = r _ { n } , }
\end{array} \quad \text { where } \quad \left\{\begin{array}{ll}
l_{k}=1-a_{k j_{k}} z_{k}, & \text { if } a_{k j_{k}} \neq 0 \\
l_{k}=1 / z_{k}, & \text { if } a_{k j_{k}}=0 .
\end{array}\right.\right.
$$

Lemma 1. For sufficiently small $r_{i}$ a global cycle $\Gamma_{h}$ defined by (5) has connected components (local cycles) in the neighborhoods of the roots $a_{J}$. Moreover, $\Gamma_{h}$ is homologous to the sum of the local cycles $\Gamma_{h, \tilde{a}_{J}}$.

Proof. Consider the global cycle $\Gamma_{h}$ defined by

$$
\left\{\begin{array}{l}
\left|h_{1}\right|=r_{1} \\
\cdots \\
\left|h_{n}\right|=r_{n}
\end{array}\right.
$$

If $a_{k j_{k}} \neq 0$ for any $k=1, \ldots, n$ in a neighborhood of $a_{J}$, then $\Gamma_{h}$ is homotopic to the cycle $\Gamma_{q, \tilde{a}_{J}}$ defined by (6) with the homotopy defined by

$$
\left\{\begin{array}{l}
\left|1-t a_{11} z_{1}\right|^{m_{11}} \cdot \ldots \cdot\left|1-a_{1 j_{1}} z_{j 1}\right|^{m_{1 j_{1}}} \cdot \ldots \cdot\left|1-t a_{1 n} z_{n}\right|^{m_{1 n}}=r_{1}, \\
\ldots \\
\left|1-t a_{n 1} z_{1}\right|^{m_{n 1}} \cdot \ldots \cdot\left|1-a_{n j_{n}} z_{j n}\right|^{m_{n j_{n}}} \cdot \ldots \cdot\left|1-t a_{n n} z_{n}\right|^{m_{n n}}=r_{n}
\end{array}\right.
$$

where $t \in[0,1]$. For $t=1$ we obtain $\Gamma_{h}$, and for $t=0$ we obtain $\Gamma_{h, \tilde{a}_{J}}$.

Now we consider $\Gamma_{h}$ in the neighborhood of $a_{J}$ where $a_{i_{1} j_{i_{1}}}=\ldots=a_{i_{k} j_{i_{k}}}=0$. Then in such neighborhood $\Gamma_{h}$ is homotopic to the cycle $\Gamma_{h, \tilde{a}_{J}}$ defined by (6), and the homotopy is defined similarly to (7) where for each $a_{i_{k} j_{i_{k}}}=0$ the corresponding term $1-a_{i_{k} j_{i_{k}}} z_{j_{i_{k}}}$ is replaced with $1 / z_{i_{k}}$.

For

$$
F_{j}(z, t)=q_{j}(z)+t \cdot Q_{j}(z), \quad j=1, \ldots, n
$$

consider the system of equations $F_{j}(z, t)=0$ which depends on a real parameter $t \geqslant 0$.

Let $r_{1}>0, \ldots, r_{n}>0$ be fixed real numbers. Compactness of the cycles $\Gamma_{h}$ defined by (5) yields the fact that for sufficiently small $t>0$, the inequalities

$$
\left|q_{j}(z)\right|>\left|t \cdot Q_{j}(z)\right|, j=1, \ldots, n
$$


hold on $\Gamma_{h}$.

By $J_{\gamma}(t)$ we denote the integral

$$
J_{\gamma}(t)=\frac{1}{(2 \pi i)^{n}} \int_{\Gamma_{h}} \frac{1}{z^{\gamma+I}} \cdot \frac{d_{z} F}{F}=\frac{1}{(2 \pi i)^{n}} \int_{\Gamma_{h}} \frac{1}{z_{1}^{\gamma_{1}+1} \ldots z_{n}^{\gamma_{n}+1}} \cdot \frac{d_{z} F_{1}}{F_{1}} \wedge \ldots \wedge \frac{d_{z} F_{n}}{F_{n}},
$$

where $\gamma=\left(\gamma_{1}, \ldots, \gamma_{n}\right)$ is a multi-index and $I=(1, \ldots, 1)$. This integral we call the residue integral in accordance with the paper [21].

We now introduce the following notations used in the theorem below and in some of the further statements.

Denote by $\Delta=\Delta(t)$ the Jacobian of the system $F_{1}(z, t), \ldots, F_{n}(z, t)$ with respect to $z_{1}, \ldots, z_{n}$ :

$$
\Delta=\operatorname{det}\left(\begin{array}{ccc}
\frac{\partial F_{1}}{\partial z_{1}} & \cdots & \frac{\partial F_{1}}{\partial z_{n}} \\
\vdots & \ddots & \vdots \\
\frac{\partial F_{n}}{\partial z_{1}} & \cdots & \frac{\partial F_{n}}{\partial z_{n}}
\end{array}\right)
$$

Recall that $\left(j_{1} \ldots j_{n}\right)$ is a permutation of $(1 \ldots n)$. By $(-1)^{s(J)}$ we denote the sign of $J$.

Let $\alpha=\left(\alpha_{1}, \ldots, \alpha_{n}\right)$ be a multi-index of length $n$. By $q^{\alpha+I}(J)$ we denote the quantity

$$
q_{1}^{\alpha_{1}+1}\left[j_{1}\right] \cdot \ldots \cdot q_{n}^{\alpha_{n}+1}\left[j_{n}\right],
$$

where $q_{s}\left[j_{s}\right]$ is the product $\left(1-a_{j 1} z_{1}\right)^{m_{j 1}} \cdot \ldots \cdot\left(1-a_{j n} z_{n}\right)^{m_{j n}}$ with the term $\left(1-a_{s j_{s}} z_{s}\right)^{m_{s j_{s}}}$ omitted:

$$
q_{s}\left[j_{s}\right]=\prod_{\substack{1 \leqslant k \leqslant n \\ k \neq s}}\left(1-a_{j k} z_{k}\right)^{m_{j k}} .
$$

By $\beta(\alpha, J)$ we denote the vector

$$
\beta(\alpha, J)=\left(m_{1 j_{1}}\left(\alpha_{j_{1}}+1\right)-1, \ldots, m_{n j_{n}}\left(\alpha_{j_{n}}+1\right)-1\right),
$$

and

$$
\beta(\alpha, J) !=\prod_{p}\left(m_{p j_{p}}\left(\alpha_{j_{p}}+1\right)-1\right) !
$$

Finally, $a_{J}^{\beta+I}$ denotes $a_{1 j_{1}}^{m_{1 j_{1}}\left(\alpha_{j_{1}}+1\right)} \cdot \ldots \cdot a_{n j_{n}}^{m_{n j_{n}}\left(\alpha_{j_{n}}+1\right)}$, and

$$
\frac{\partial^{\| \beta(\alpha(J) \|}}{\partial z^{\beta(\alpha, J)}}=\frac{\partial^{m_{1 j_{1}}\left(\alpha_{j_{1}}+1\right)-1+\ldots+m_{n j_{n}}\left(\alpha_{j_{n}}+1\right)-1}}{\partial z_{1}^{m_{1 j_{1}}\left(\alpha_{j_{1}}+1\right)-1} \ldots \partial z_{n}^{m_{n j_{n}}\left(\alpha_{j_{n}}+1\right)-1}}
$$

is a differentiation operator.

Theorem 2. Under the assumptions made for the functions $F_{i}$ defined by (8), the following formulas for $J_{\gamma}(t)$ as convergent (for sufficiently small $t$ ) series are valid:

$$
\begin{aligned}
J_{\gamma}(t)=\sum_{J}^{\prime} \sum_{\alpha}(-t)^{\|\alpha\|+\| \beta(\alpha, J) \mid+n} \frac{(-1)^{s(J)}}{\beta(\alpha, J) ! \cdot a_{J}^{\beta+I}} & \\
& \times \frac{\partial^{\| \beta(\alpha(J) \|}}{\partial z^{\beta(\alpha, J)}}\left[\frac{\Delta(t)}{z_{1}^{\gamma_{1}+1} \cdot \ldots \cdot z_{n}^{\gamma_{n}+1}} \cdot \frac{Q^{\alpha}}{q^{\alpha+I}(J)}\right]_{z=\tilde{a}_{J}},
\end{aligned}
$$

where $\sum_{J}^{\prime}$ means that the summation is performed over all multi-indices $J$ such that $a_{J}$ have no zero components. 
Proof. We have

$$
\begin{aligned}
J_{\gamma}(t)=\frac{1}{(2 \pi i)^{n}} \int_{\Gamma_{h}} \frac{1}{z^{\gamma+I}} \cdot \frac{d F}{F} & =\frac{1}{(2 \pi i)^{n}} \int_{\Gamma_{h}} \frac{1}{z_{1}^{\gamma_{1}+1} \cdot \ldots \cdot z_{n}^{\gamma_{n}+1}} \cdot \frac{d F_{1}}{F_{1}} \wedge \ldots \wedge \frac{d F_{n}}{F_{n}} \\
& =\frac{1}{(2 \pi i)^{n}} \int_{\Gamma_{h}} \frac{1}{z^{\gamma+I}} \cdot \frac{\Delta d z}{F},
\end{aligned}
$$

where $d z=d z_{1} \wedge \ldots \wedge d z_{n}$, and $F=F_{1} \cdot \ldots \cdot F_{n}$.

Applying a formula for the sum of a geometric series, we get

$$
\begin{aligned}
& \frac{1}{(2 \pi i)^{n}} \int_{\Gamma_{h}} \frac{1}{z_{1}^{\gamma_{1}+1} \cdot \ldots \cdot z_{n}^{\gamma_{n}+1}} \cdot \frac{\Delta d z}{F_{1} \cdot \ldots \cdot F_{n}} \\
& =\frac{1}{(2 \pi i)^{n}} \sum_{\|\alpha\| \geqslant 0}(-t)^{\|\alpha\|} \int_{\Gamma_{h}} \frac{\Delta(t)}{z_{1}^{\gamma_{1}+1} \cdot \ldots \cdot z_{n}^{\gamma_{n}+1}} \cdot \frac{Q_{1}^{\alpha_{1}} \cdot \ldots \cdot Q_{n}^{\alpha_{n}}}{q_{1}^{\alpha_{1}+1} \cdot \ldots \cdot q_{n}^{\alpha_{n}+1}} d z \\
& =\frac{1}{(2 \pi i)^{n}} \sum_{J} \sum_{\|\alpha\| \geqslant 0}(-t)^{\|\alpha\|} \int_{\Gamma_{\Gamma_{h, \tilde{a}_{J}}}} \frac{\Delta(t)}{z_{1}^{\gamma_{1}+1} \cdot \ldots \cdot z_{n}^{\gamma_{n}+1}} \cdot \frac{Q_{1}^{\alpha_{1}} \cdot \ldots \cdot Q_{n}^{\alpha_{n}}}{q_{1}^{\alpha_{1}+1} \cdot \ldots \cdot q_{n}^{\alpha_{n}+1}} d z \\
& =\frac{1}{(2 \pi i)^{n}} \sum_{J}^{\prime} \sum_{\|\alpha\| \geqslant 0}(-t)^{\|\alpha\|} \int_{\Gamma_{\Gamma_{h, \tilde{a}_{J}}}} \frac{\Delta(t)}{z_{1}^{\gamma_{1}+1} \cdot \ldots \cdot z_{n}^{\gamma_{n}+1}} \cdot \frac{Q_{1}^{\alpha_{1}} \cdot \ldots \cdot Q_{n}^{\alpha_{n}}}{q_{1}^{\alpha_{1}+1} \cdot \ldots \cdot q_{n}^{\alpha_{n}+1}} d z \\
& =\frac{1}{(2 \pi i)^{n}} \sum_{J}^{\prime}(-1)^{s(J)} \sum_{\|\alpha\| \geqslant 0}(-t)^{\left\|\alpha^{s}\right\|_{X}} \\
& \times \int_{\Gamma_{h, \tilde{a}_{J}}} \frac{\Delta(t)}{z^{\gamma+I}} \cdot \frac{Q^{\alpha} d z}{q_{1}^{\alpha_{1}+1}\left[j_{1}\right] \ldots q_{n}^{\alpha_{n}+1}\left[j_{n}\right]\left(1-a_{1 j_{1}} z_{j_{1}}\right)^{\left(\alpha_{1}+1\right) m_{1 j_{1}}} \ldots\left(1-a_{n j_{n}} z_{j_{n}}\right)^{\left(\alpha_{n}+1\right) m_{n j_{n}}}},
\end{aligned}
$$

and finally we obtain that $J_{\gamma}(t)$ is equal to

$$
\sum_{J}^{\prime} \sum_{\alpha}(-t)^{\|\alpha\|+\|\beta\|+n} \frac{(-1)^{s(J)}}{\beta(\alpha, J) ! \cdot a_{J}^{\beta+I}} \cdot \frac{\partial^{\|\beta(\alpha, J)\|}}{\partial z^{\beta(\alpha, J)}}\left[\frac{\Delta(t)}{z_{1}^{\gamma_{1}+1} \cdot \ldots \cdot z_{n}^{\gamma_{n}+1}} \cdot \frac{Q^{\alpha}}{q^{\alpha+I}(J)}\right]_{z=\tilde{a}_{J}} .
$$

The resulting series converges for sufficiently small $t$.

\section{Residue integrals and Waring's formulas for algebraic systems}

In this section we establish a correspondence between the residue integrals and the power sums of the inverses to the roots (Waring's formulas). First we shrink the class of systems for which

the sums in Theorem 2 are finite. Then, applying the transformations $z_{j}=\frac{1}{w_{j}}, j=1, \ldots, n$, and 
Lemma 4 by A. Tsikh we rewrite the residue integrals $J_{\gamma}(t)$ in the new variables $w$ (Lemma 3 ). Further, Lemma 5 shows that $J_{\gamma}(t)$ can be expressed by a finite number of Taylor coefficients of the considered functions. Theorem 7 shows (by means of Lemma 6) that the residue integral $J_{\gamma}(t)$ equals (up to a sign) to the power sums of the inverses to the roots. The main result of the paper, Theorem 8, shows that the statement of Theorem 7 is true not only for sufficiently small $t>0$ but also for $t=1$. (Note that Theorems 8 and 9 allow one find power sums of the inverses to the roots of the systems without finding the roots.) As a conclusion of the section we present an elimination method for the considered systems.

Suppose

$$
Q_{j}(z)=z_{1} \cdot \ldots \cdot z_{n} \sum_{\mid \alpha \| \geq 0} C_{\alpha}^{j} z^{\alpha} \quad j=1, \ldots, n,
$$

where $\alpha$ is a multi-index, $z^{\alpha}=z_{1}^{\alpha_{1}} \cdots z_{n}^{\alpha_{n}}$, and $\operatorname{deg}_{z_{k}} Q_{j} \leqslant m_{j k}, j, k=1, \ldots, n$ for all non-zero $a_{j k}$. If $a_{j k}=0$ then no restriction on $\operatorname{deg}_{z_{k}} Q_{j}$ is needed.

Assuming that all $w_{j} \neq 0$, we substitute $z_{j}=\frac{1}{w_{j}}, j=1, \ldots, n$ in the functions

$$
F_{j}(z, t)=\left(q_{j}(z)+t \cdot Q_{j}(z)\right), \quad j=1, \ldots, n
$$

Consequently, for $j=1, \ldots, n$, we get

$$
\begin{gathered}
F_{j}\left(\frac{1}{w_{1}}, \ldots, \frac{1}{w_{n}}, t\right)=q_{j}\left(\frac{1}{w_{1}}, \ldots, \frac{1}{w_{n}}\right)+t \cdot Q_{j}\left(\frac{1}{w_{1}}, \ldots, \frac{1}{w_{n}}\right) \\
=\left(1-a_{j 1} \frac{1}{w_{1}}\right)^{m_{j 1}} \cdots\left(1-a_{j n} \frac{1}{w_{n}}\right)^{m_{j n}}+t \cdot Q_{j}\left(\frac{1}{w_{1}}, \ldots, \frac{1}{w_{n}}\right) \\
=\left(\frac{1}{w_{1}}\right)^{m_{j 1}} \cdots\left(\frac{1}{w_{n}}\right)^{m_{j n}} \cdot\left(w_{1}-a_{j 1}\right)^{m_{j 1}} \cdot \ldots \cdot\left(w_{n}-a_{j n}\right)^{m_{j n}}+t \cdot Q_{j}\left(\frac{1}{w_{1}}, \ldots, \frac{1}{w_{n}}\right) .
\end{gathered}
$$

And finally we arrive at

$$
F_{j}\left(\frac{1}{w_{1}}, \ldots, \frac{1}{w_{n}}, t\right)=\left(\frac{1}{w_{1}}\right)^{m_{j 1}} \ldots\left(\frac{1}{w_{n}}\right)^{m_{i n}} \cdot\left(\widetilde{q}_{j}(w)+t \cdot \widetilde{Q}_{j}(w)\right),
$$

where $\widetilde{q}_{j}$ are the functions

$$
\widetilde{q}_{j}=\left(w_{1}-a_{j 1}\right)^{m_{j 1}} \ldots\left(w_{n}-a_{j n}\right)^{m_{j n}}
$$

$\widetilde{Q}_{j}$ are the polynomials

$$
\widetilde{Q}_{j}=w_{1}^{m_{j 1}} \ldots w_{n}^{m_{j n}} \cdot Q_{j}\left(\frac{1}{w_{1}}, \ldots, \frac{1}{w_{n}}\right)
$$

and

$$
\widetilde{F}_{j}=\widetilde{F}_{j}(w, t)=\widetilde{q}_{j}(w)+t \cdot \widetilde{Q}_{j}(w), \quad j=1, \ldots, n .
$$

From (10) we obtain

$$
\operatorname{deg}_{w_{j}} \widetilde{Q}_{j}<m_{j k}, j, k=1, \ldots, n
$$


Note that in the above calculations it is not important whether $a_{j k}$ vanish or not. Indeed, suppose that in $F_{j}(z, t)=q_{j}(z)+t \cdot Q_{j}(z), j=1, \ldots, n$, some $a_{j k}=0$. If, for instance, $a_{11}=0$, then after the substitution $z_{j}=\frac{1}{w_{j}}, j=1, \ldots, n$, the function $F_{1}$ takes the form

$$
F_{1}\left(\frac{1}{w_{1}}, \ldots, \frac{1}{w_{n}}, t\right)=q_{1}\left(\frac{1}{w_{1}}, \ldots, \frac{1}{w_{n}}\right)+t \cdot Q_{1}\left(\frac{1}{w_{1}}, \ldots, \frac{1}{w_{n}}\right),
$$

where

$$
\begin{aligned}
& q_{1}\left(\frac{1}{w_{1}}, \ldots, \frac{1}{w_{n}}\right)=(1\left.-a_{12} \frac{1}{w_{2}}\right)^{m_{12}} \cdot \ldots \cdot\left(1-a_{1 n} \frac{1}{w_{n}}\right)^{m_{1 n}} \\
&=\left(\frac{1}{w_{1}}\right)^{\operatorname{deg}_{w_{1}} Q_{1}} \cdot\left(\frac{1}{w_{2}}\right)^{m_{12}} \cdot \ldots \cdot\left(\frac{1}{w_{n}}\right)^{m_{1 n}} \\
& \quad \times w_{1}^{\operatorname{deg}_{w_{1}} Q_{1}} \cdot\left(w_{2}-a_{12}\right)^{m_{12}} \cdot \ldots \cdot\left(w_{n}-a_{1 n}\right)^{m_{1 n}} .
\end{aligned}
$$

Consequently

$$
F_{1}\left(\frac{1}{w_{1}}, \ldots, \frac{1}{w_{n}}, t\right)=\left(\frac{1}{w_{1}}\right)^{\operatorname{deg}_{w_{1}} Q_{1}} \cdot \ldots \cdot\left(\frac{1}{w_{n}}\right)^{m_{1 n}} \cdot\left(\widetilde{q}_{1}(w)+t \cdot \widetilde{Q}_{1}(w)\right),
$$

where

$$
\widetilde{q}_{1}=\left(w_{1}\right)^{\operatorname{deg}_{w_{1}} Q_{1}} \cdot\left(w_{2}-a_{12}\right)^{m_{12}} \cdot \ldots \cdot\left(w_{n}-a_{1 n}\right)^{m_{1 n}},
$$

and

$$
\widetilde{Q}_{1}=w_{1}^{\operatorname{deg}_{w_{1}} Q_{1}} \cdot w_{2}^{m_{12}} \cdot \ldots \cdot w_{n}^{m_{1 n}} \cdot Q_{1}\left(\frac{1}{w_{1}}, \ldots, \frac{1}{w_{n}}\right) .
$$

So, we can take $m_{11}=\operatorname{deg}_{w_{1}} Q_{1}$. From (10) we derive that

$$
\operatorname{deg}_{w_{j}} \widetilde{Q}_{1}<m_{1 j}, j=1, \ldots, n .
$$

When $0 \leqslant t<<1$, the system (12) has finite number of zeros in $\mathbb{C}^{n}$ which depend on $t$. Moreover, (12) does not have infinite roots in $\left(\mathbb{P}^{1}(\mathbb{C})\right)^{n}$ (see [23] and $[5$, Theorems 8.5, 8.6]). As was shown in [23] (see also [5, Theorem 8.5]) the number of zeros (counting multiplicities) is equal to the permanent of the matrix $\left(m_{i j}\right)_{1 \leqslant i, j \leqslant n}$.

Consider the cycle

$$
\widetilde{\Gamma}_{h}=\left\{w \in \mathbb{C}^{n}:\left|h_{j}\left(\frac{1}{w_{1}}, \ldots, \frac{1}{w_{n}}\right)\right|=\varepsilon_{j}, \quad j=1, \ldots, n\right\} .
$$

Compactness of the cycle $\widetilde{\Gamma}_{h}$ implies that

$$
\left|q_{j}\left(\frac{1}{w_{1}}, \ldots, \frac{1}{w_{n}}\right)\right|>\left|t \cdot Q_{j}\left(\frac{1}{w_{1}}, \ldots, \frac{1}{w_{n}}\right)\right|, \quad j=1, \ldots, n
$$

for $t$ small enough.

Therefore, $\widetilde{\Gamma}_{h}$ is homologous to the sum of the cycles $\widetilde{\Gamma}_{h, \tilde{a}_{J}}$

$$
\left\{\begin{array}{l}
\left|1-a_{1 j_{1}} \frac{1}{w_{1}}\right|=\varepsilon_{1}, \\
\cdots \\
\left|1-a_{n j_{n}} \frac{1}{w_{n}}\right|=\varepsilon_{n},
\end{array}\right.
$$


obtained from the cycles $\Gamma_{h, \tilde{a}_{J}}$ by the substitution $z_{j}=\frac{1}{w_{j}}$.

The equation

$$
\left|1-a_{j k_{j}} \frac{1}{w_{j}}\right|=\varepsilon
$$

defines a circle. Indeed, let us first rewrite it in the form

$$
\left|w_{j}-a_{j k_{j}}\right|=\varepsilon\left|w_{j}\right| \quad \text { or } \quad\left|w_{j}-a_{j k j}\right|^{2}=\varepsilon^{2}\left|w_{j}\right|^{2} .
$$

Thus

or

$$
\left(1-\varepsilon^{2}\right)\left|w_{j}-\frac{a_{j k_{j}}}{1-\varepsilon^{2}}\right|^{2}=\frac{\varepsilon^{2} \cdot\left|a_{j k_{j}}\right|^{2}}{\left(1-\varepsilon^{2}\right)}
$$

$$
\left|w_{j}-\frac{a_{j k_{j}}}{1-\varepsilon^{2}}\right|^{2}=\frac{\varepsilon^{2} \cdot\left|a_{j k_{j}}\right|^{2}}{\left(1-\varepsilon^{2}\right)^{2}}, \quad j=1, \ldots, n .
$$

For sufficiently small $\varepsilon$ the point $a_{j k_{j}}$ lies inside this circle, and, therefore, $\widetilde{\Gamma}_{h, \tilde{a}_{J}}$ is homologous to the cycle $\widetilde{\Gamma}_{h, a_{J}}$ :

$$
\left\{\begin{array}{l}
\left|w_{1}-a_{1 j_{1}}\right|=\varepsilon_{1}, \\
\cdots \\
\left|w_{n}-a_{n j_{n}}\right|=\varepsilon_{n} .
\end{array}\right.
$$

Here $a_{k j_{k}}$ can vanish for some $k$.

Lemma 3. The following formula holds for the residue integral (9):

$$
J_{\gamma}(t)=\frac{(-1)^{n}}{(2 \pi i)^{n}} \int_{\widetilde{\Gamma}_{h}} w_{1}^{\gamma_{1}+1} \cdot \ldots \cdot w_{n}^{\gamma_{n}+1} \cdot \frac{d \widetilde{F}_{1}}{\widetilde{F}_{1}} \wedge \ldots \wedge \frac{d \widetilde{F}_{n}}{\widetilde{F}_{n}}
$$

Proof. The equality (11) yields

$$
F_{j}\left(\frac{1}{w_{1}}, \ldots, \frac{1}{w_{n}}, t\right)=\left(\frac{1}{w_{1}}\right)^{m_{i 1}} \cdot \ldots \cdot\left(\frac{1}{w_{n}}\right)^{m_{i n}} \cdot \widetilde{F}_{j}(w, t), \quad j=1, \ldots, n .
$$

Then

$$
\frac{d F_{j}\left(\frac{1}{w_{1}}, \frac{1}{w_{2}}, \ldots, \frac{1}{w_{n}}, t\right)}{F_{j}\left(\frac{1}{w_{1}}, \frac{1}{w_{2}}, \ldots, \frac{1}{w_{n}}, t\right)}=\frac{d \widetilde{F}_{j}(w, t)}{\widetilde{F}_{j}(w, t)}-\sum_{k=1}^{n} m_{j k} \cdot \frac{d w_{k}}{w_{k}}
$$

Using (11) and taking into account the change of orientation of the space after replacing $z_{j}=1 / w_{j}, j=1, \ldots, n$, one can rewrite the integral $J_{\gamma}(t)$ as

$$
\begin{gathered}
J_{\gamma}(t)=\frac{(-1)^{n}}{(2 \pi i)^{n}} \int_{\widetilde{\Gamma}_{h}} w^{\gamma+I} \cdot \frac{d F\left(\frac{1}{w_{1}}, \ldots, \frac{1}{w_{n}}, t\right)}{F\left(\frac{1}{w_{1}}, \ldots, \frac{1}{w_{n}}, t\right)} \\
=\frac{(-1)^{n}}{(2 \pi i)^{n}} \int_{\widetilde{\Gamma}_{h}} w_{1}^{\gamma_{1}+1} \ldots w_{n}^{\gamma_{n}+1} \cdot \frac{d F_{1}}{F_{1}} \wedge \ldots \wedge \frac{d F_{n}}{F_{n}} \\
=\frac{(-1)^{n}}{(2 \pi i)^{n}} \int_{\widetilde{\Gamma}_{h}} w^{\gamma+I}\left(\frac{d \widetilde{F}_{1}(w)}{\widetilde{F}_{1}(w)}-\sum_{k=1}^{n} m_{1 k} \cdot \frac{d w_{k}}{w_{k}}\right) \wedge \ldots \wedge\left(\frac{d \widetilde{F}_{n}(w)}{\widetilde{F}_{n}(w)}-\sum_{k=1}^{n} m_{n k} \cdot \frac{d w_{k}}{w_{k}}\right) .
\end{gathered}
$$


All the integrals

$$
\int_{\widetilde{\Gamma}_{h}} w^{\gamma+I} \frac{d \widetilde{F}_{i_{1}}(w)}{\widetilde{F}_{i_{1}}(w)} \wedge \ldots \wedge \frac{d \widetilde{F}_{i_{l}}(w)}{\widetilde{F}_{i_{l}}(w)} \wedge \frac{d w_{j_{1}}}{w_{j_{1}}} \wedge \ldots \wedge \frac{d w_{j_{n-l}}}{w_{j_{n-l}}}
$$

vanish when $0 \leqslant l<n$ and $\varepsilon_{j}$ are large enough.

Indeed, when $\varepsilon_{j}, j=1, \ldots, n$ are sufficiently large, the inequalities

$$
\left|\widetilde{q}_{j}\right|>\left|t \cdot \widetilde{Q}_{j}(w)\right|
$$

hold on $\widetilde{\Gamma}_{h}$. Therefore

$$
\frac{1}{\widetilde{F}_{j}(w)}=\sum_{p=0}^{\infty} \frac{(-1)^{p} t^{p} \widetilde{Q}_{j}^{p}(w)}{\widetilde{q}_{j}^{(p+1)}} .
$$

Consequently, the integrals (13) are absolutely convergent series of the integrals

$$
\int_{\widetilde{\Gamma}_{h}} w^{\gamma+I} \frac{w^{\alpha} d w_{1} \wedge \ldots \wedge d w_{n}}{\widetilde{q}_{1}^{\left(p_{1}+1\right)} \ldots \widetilde{q}_{i_{l}}^{\left(p_{l}+1\right)} \cdot w_{j_{1}} \ldots w_{j_{n-l}}} .
$$

Stokes' theorem and the fact that all the integrands are holomorphic imply vanishing of all these integrals.

Finally, we arrive at

$$
J_{\gamma}(t)=\frac{(-1)^{n}}{(2 \pi i)^{n}} \int_{\widetilde{\Gamma}_{h}} w_{1}^{\gamma_{1}+1} \ldots w_{n}^{\gamma_{n}+1} \cdot \frac{d \widetilde{F}_{1}}{\widetilde{F}_{1}} \wedge \ldots \wedge \frac{d \widetilde{F}_{n}}{\widetilde{F}_{n}}
$$

Now we state a result from [23] that we will need for further discussion.

Consider a system of algebraic equations in $\mathbb{C}^{n}$ :

$$
f_{j}(z)=0, \quad j=1, \ldots, n \text {. }
$$

Suppose (15) has finite number of roots in $\mathbb{C}^{n}$ and does not have infinite roots in $\left(\mathbb{C P}^{1}\right)^{n}$.

Denote $m_{j k}=\operatorname{deg}_{z_{k}} f_{j}$. When $r_{1}, \ldots, r_{n}$ are sufficiently small, the cycle

$$
\Gamma=\left\{z \in \mathbb{C}^{n}:\left|f_{1}(z)\right|=r_{1}, \ldots,\left|f_{n}(z)\right|=r_{n}\right\}
$$

is homologous to the sum of cycles lying in the neighborhood of the roots of (15).

Lemma 4 (A. Tsikh, [23]). Under the above assumptions

$$
\int_{\Gamma} \frac{P(z) d z}{f_{1}(z) \ldots f_{n}(z)}=0
$$

for any polynomial $P(z)$ such that $p_{j}=\operatorname{deg}_{z_{j}} P \leqslant m_{1 j}+\ldots+m_{n j}$ for all $j=1, \ldots, n$.

The Lemma was proved using the residue theorem (theorem on a total sum of residues) on a compact complex manifold, namely for example a smooth toric compactification of $\mathbb{T}^{n}$. 
Lemma 5. Let $\widetilde{\Delta}=\widetilde{\Delta}(w, t)$ be the Jacobian of (12) with respect to $w_{1}, \ldots, w_{n}$. Then

$$
J_{\gamma}(t)=\sum_{K \in \Re}(-t)^{\|K\|+n} \sum_{J} \frac{(-1)^{s(J)}}{\beta(K, J) !} \cdot \frac{\partial^{\|\beta(K, J)\|}}{\partial w^{\beta}(K, J)}\left[\widetilde{\Delta} \cdot w_{1}^{\gamma_{1}+1} \cdots w_{n}^{\gamma_{n}+1} \cdot \frac{\widetilde{Q}^{K}}{\widetilde{q}^{K+I}(J)}\right]_{w=a_{J}},
$$

where $K=\left(k_{1}, \ldots, k_{n}\right)$ is multi-index, $\widetilde{Q}^{K}=\widetilde{Q}_{1}^{k_{1}} \cdot \ldots \cdot \widetilde{Q}_{n}^{k_{n}}$,

$$
\begin{gathered}
\beta(K, J)=\left(m_{1 j_{1}}\left(k_{j_{1}}+1\right)-1, \ldots, m_{n j_{n}}\left(k_{j_{n}}+1\right)-1\right), \\
\beta(K, J) !=\prod_{p}\left(m_{p j_{p}}\left(k_{j_{p}}+1\right)-1\right) ! \text {, and } \\
\Re=\left\{K=\left(k_{1}, \ldots, k_{n}\right) \text { : there exists } \gamma_{j} \text { such that }\|K\|<\gamma_{j}+2, j=1, \ldots, n\right\} .
\end{gathered}
$$

The rest of the notations in the statement are as in Theorem 2.

Proof. The proof of (16) completely repeats the proof of the formula for $J_{\gamma}(t)$ in Theorem 2 except for the finiteness of the sum.

We now show that the summation in the above formulas is over a finite set of multiindices. To show this, we estimate the degrees in $w_{j}$ of the numerator and compare with the corresponding degrees of the denominator of (16).

The degree of the numerator in $w_{j}$ is less than or equal to

$$
p_{j}=m_{1 j}+\ldots+m_{n j}-1+\gamma_{j}+1+\left(m_{1 j}-1\right) k_{1}+\ldots+\left(m_{n j}-1\right) k_{n} .
$$

The corresponding degree of the denominator is

$$
s_{j}=m_{1 j}\left(k_{1}+1\right)+\ldots+m_{n j}\left(k_{n}+1\right) .
$$

Lemma 4 implies vanishing of all the integrals for which the inequality $p_{i} \leqslant s_{i}-2$ holds for all $j=1, \ldots, n$, so that

$$
\begin{aligned}
m_{1 j}+\ldots+m_{n j}-1+\gamma_{j}+1+\left(m_{1 j}-1\right) k_{1}+\ldots+ & \left(m_{n j}-1\right) k_{n} \\
& \leqslant m_{1 j}\left(k_{1}+1\right)+\ldots+m_{n j}\left(k_{n}+1\right)-2 .
\end{aligned}
$$

After combining similar terms we arrive at

$$
\gamma_{j}+1-k_{1}-\ldots-k_{n}-1 \leqslant-2
$$

SO

$$
\gamma_{j}+2 \leqslant\|K\|
$$

Thus, the only non-zero integrals in (16) are the ones for which $K$ runs over such set that $\gamma_{j}+2>\|K\|$ for at least one $\gamma_{j}$.

Lemma 6. Let $w_{1}, \ldots, w_{s}$ where $w_{j}=\left(w_{j 1}, \ldots, w_{j n}\right), j=1, \ldots, n$ be all the zeros (depending on t) of (12) counting multiplicities. Then

$$
J_{\gamma}=(-1)^{n} \sum_{j=1}^{s} w_{j 1}^{\gamma_{1}+1} \cdot w_{j 2}^{\gamma_{2}+1} \cdots w_{j n}^{\gamma_{n}+1}
$$


The number $s$ of the zeros is equal to the permanent of the matrix $\left(m_{i j}\right)_{1 \leqslant i, j \leqslant n}$ (see [23] or $[5$, Theorem 8.5]).

Proof. The statement follows from the multidimensional logarithmic residue formula and the theorem on shifted skeleton (see [3, Chapter 3]).

Denote by $z^{(k)}(t)=\left(z_{k 1}(t), \ldots, z_{k n}(t)\right), k=1, \ldots, s$, the zeros of $(2)$ with the functions $t Q_{j}$, where $Q_{j}$ are defined by (10). The number of the zeros of this system in $\mathbb{C}^{n}$ is finite (see Section 2). Denote by $p$ the number of zeros (multiplicities taken into account) in $\mathbb{T}^{n}$, that is in $\mathbb{C}^{n}$ minus the coordinate axes. Since $z^{(k)}$ do not lie in coordinate subspaces, then $z_{k m}=1 / w_{k m}$, $m=1, \ldots, n$ and therefore we have finite number $p$ of the zeros, $p \leqslant s$.

Recall that $\widetilde{q}_{j}$ are the functions

$$
\widetilde{q}_{j}=\left(w_{1}-a_{j 1}\right)^{m_{j 1}} \cdots\left(w_{n}-a_{j n}\right)^{m_{j n}}
$$

and $\widetilde{Q}_{j}$ are the polynomials

$$
\widetilde{Q}_{j}=w_{1}^{m_{j 1}} \cdots w_{n}^{m_{j n}} \cdot Q_{j}\left(\frac{1}{w_{1}}, \ldots, \frac{1}{w_{n}}\right) .
$$

Theorem 7. The following equality holds:

$$
\begin{gathered}
\sum_{j=1}^{p} \frac{1}{z_{k 1}(t)^{\gamma_{1}+1} \cdots z_{k n}(t)^{\gamma_{n}+1}} \\
=\sum_{K \in \Re}(-t)^{\|K\|+n} \sum_{J} \frac{(-1)^{s(J)}}{\beta(K, J) !} \cdot \frac{\partial^{\|\beta(K, J)\|}}{\partial w^{\beta}(K, J)}\left[\widetilde{\Delta}(t) \cdot w_{1}^{\gamma_{1}+1} \cdots w_{n}^{\gamma_{n}+1} \cdot \frac{\widetilde{Q}^{K}}{\widetilde{q}^{K+I}(J)}\right]_{w=a_{J}} .
\end{gathered}
$$

Proof. The statement follows from Lemmas 5 and 6 . Here we use the notations of Theorem 2, with the corresponding changes.

Thus, the power sum of zeros of (12) is a polynomial on $t$, and, therefore, the equality in Theorem 7 also holds for $t=1$.

Denote

$$
\sigma_{\gamma+I}=\sum_{k=1}^{p} \frac{1}{z_{k 1}^{\gamma_{1}+1} \cdots z_{k n}^{\gamma_{n}+1}},
$$

where $z^{(k)}=\left(z_{k 1}, \ldots, z_{k n}\right)=\left(z_{k 1}(1), \ldots, z_{k n}(1)\right), k=1, \ldots, p$.

Theorem 8 (Waring's formulas). For the system $f_{j}=0, j=1, \ldots, n$, with functions $f_{j}$ defined by (2) and $Q_{i}$ defined by (10) the following formulas are valid:

$$
\begin{gathered}
\sigma_{\gamma+I}=\sum_{k=1}^{p} \frac{1}{z_{j 1}^{\gamma_{1}+1} \cdots z_{j n}^{\gamma_{n}+1}} \\
=\frac{1}{(2 \pi i)^{n}} \sum_{\|K\| \geqslant 0}(-1)^{\|K\|+n} \sum_{J}(-1)^{s(J)} \int_{\widetilde{\Gamma}_{h, a}} \widetilde{\Delta} \cdot w_{1}^{\gamma_{1}+1} \cdots w_{n}^{\gamma_{n}+1} \cdot \frac{\widetilde{Q}_{1}^{k_{1}} \cdots \widetilde{Q}_{n}^{k_{n}}}{\widetilde{q}_{1}^{k_{1}+1} \cdots \widetilde{q}_{n}^{k_{n}+1}} d w \\
=\sum_{K \in \Re}(-1)^{\|K\|+n} \sum_{J} \frac{(-1)^{s(J)}}{\beta(K, J) !} \cdot \frac{\partial^{\|\beta(K, J)\|}}{\partial w^{\beta}(K, J)}\left[\widetilde{\Delta} \cdot w_{1}^{\gamma_{1}+1} \cdots w_{n}^{\gamma_{n}+1} \cdot \frac{\widetilde{Q}^{K}}{\widetilde{q}^{K+I}(J)}\right]_{w=a_{J}} .
\end{gathered}
$$


Proof. The statement of the Theorem is a corollary of Theorem 7 .

Note that in [10] the authors considered algebraic systems and obtained expansions of their solutions into geometric series. Moreover, the authors obtained analogs of Waring's formulas for the systems

$$
y_{j}^{m_{j}}+\sum_{\lambda \in \Lambda^{(j)} \cup\{0\}} x_{\lambda}^{(j)} y^{\lambda}=0, \quad \lambda_{1}+\ldots+\lambda_{n}<m_{j}, \quad j=1, \ldots, n,
$$

where leading homogeneous parts are monomials (here $\Lambda^{(j)}$ is a finite set of multi-indices).

\section{Residue integrals and Waring's formulas for transcen- dental systems}

We now consider a more general situation. Let $f_{j}$ be entire functions in $\mathbb{C}^{n}$ of finite order not greater than $\rho$ and

$$
f_{j}(z)=\prod_{m,=1}^{\infty} f_{j, m}(z), \quad j=1, \ldots, n .
$$

Here $f_{j, m}(z)$ are entire functions in $\mathbb{C}^{n}$ of finite order not greater than $\rho$ admitting expansion into uniformly convergent in $\mathbb{C}^{n}$ infinite products with factors of the form

$$
f_{j, m}(z)=\left(q_{j, m}+Q_{j, m}(z)\right),
$$

where $q_{j, m}(z)$ and $Q_{j, m}(z)$ are the polynomials defined by (3) and (10) respectively. An entire function of several complex variables is not always decomposable into an infinite product of the functions associated with its zeros (see, e.g., [19]). One could find the sufficient conditions for the existence of such an expansion (in the form of convergence of the distances between the origin and zero sets of the functions $\left.q_{j, m}+Q_{j, m}(z)\right)$ in [20].

Denote by $z^{(j)}=\left(z_{j 1}, \ldots, z_{j n}\right), j=1, \ldots, \infty$, the zeros of (17) not lying in the coordinate subspaces, counting multiplicities.

We now give a multidimensional Waring's formula for transcendental systems.

Theorem 9. Consider the system $f_{j}(z)=0, j=1, \ldots, n$, with the functions $f_{j}$ defined by (17). Then, the following formulas are valid:

$$
\begin{gathered}
\sigma_{\gamma+I}=\sum_{j=1}^{\infty} \frac{1}{z_{j 1}^{\gamma_{1}+1} \cdots z_{j n}^{\gamma_{n}+1}} \\
=\sum_{K \in \Re}(-1)^{\|K\|+n} \sum_{S} \sum_{J} \frac{(-1)^{s(J)}}{\beta(K, J) !} \cdot \frac{\partial^{\|\beta(K, J)\|}}{\partial w^{\beta(K, J)}}\left[\widetilde{\Delta} \cdot w_{1}^{\gamma_{1}+1} \cdots w_{n}^{\gamma_{n}+1} \cdot \frac{\widetilde{Q}^{K}(s)}{\widetilde{q}^{K+I}(J, s)}\right]_{w=a_{J}},
\end{gathered}
$$

where $\widetilde{Q}^{K}(s)=\widetilde{Q}_{1, s}^{k_{1}} \cdots \widetilde{Q}_{n, s}^{k_{n}}$.

Proof. We have

$$
\frac{d f_{j}(z)}{f_{j}(z)}=\frac{d \prod_{s=1}^{\infty} f_{j s}(z)}{\prod_{s=1}^{\infty} f_{j s}(z)}=\sum_{s=1}^{\infty} \frac{d f_{j s}(z)}{f_{j s}(z)} .
$$


The above series converges uniformly on $\widetilde{\Gamma}_{h, a_{J}}$. Thus, $J_{\gamma}(1)$ is defined and is equal to the convergent series of the integrals

$$
\frac{1}{(2 \pi i)^{n}} \int_{\Gamma_{q}} \frac{1}{z^{\gamma+I}} \cdot \frac{d f_{1, s_{1}}(z)}{f_{1 s_{1}}(z)} \wedge \ldots \wedge \frac{d f_{n s_{n}}(z)}{f_{n, s_{n}}(z)}
$$

where the summation is taken over the cubes with integer sides, centered at the origin. And, for each such integral the required formula was proved in Theorem 8.

If $\prod_{s=1}^{\infty} f_{j, s}(z)$ converge absolutely, then their values does not depend on the permutation of their factors. In other words, changing the numbering of the roots does not affect the values of the infinite products of $f_{j, s}(z)$. Consequently, $J_{\gamma}$ also does not depend on the permutation of its terms. This yields absolute convergence of $J_{\gamma}$ and $\sigma_{\gamma+I}$.

Remark 10. We are now ready to describe the scheme of elimination of unknowns. Consider a system of equations of the form as in Theorems 8 and 9. Let $s_{j}$ be the power sums of its roots

$$
s_{j}=\sigma_{j, \ldots, j}=\sum_{k=1}^{\infty} \frac{1}{z_{k 1}^{j} \cdot \ldots \cdot z_{k n}^{j}}, \quad j \geqslant 1 .
$$

Now, we need to find an entire function $f(w)$ of a single variable $w \in \mathbb{C}$, such that the power sums of its roots coincide with $s_{i}$ (by the Weierstrass theorem). Let the Taylor expansion of this function be

$$
f(w)=1+b_{1} w+\ldots+b_{k} w^{k}+\ldots
$$

Since the series in the right hand side of (18) converge absolutely, then $f$ can be decomposed into an infinite product with respect to its zeros

$$
c_{j}=\frac{1}{z_{k 1}^{j} \cdot \ldots \cdot z_{k n}^{j}}, j \geqslant 1
$$

(Hadamard's formula), which yields that $f(w)$ is an entire function of at most first order of growth. The analogs of recurrent Newton formulas connecting the coefficients $b_{k}$ and the sums $s_{i}$ for such functions were given in [5, Chapter 1]. More precisely, Theorem 2.3 in [5] states that

$$
\sum_{j=0}^{k-1} b_{j} s_{k-j}+k b_{k}=0, \quad b_{0}=1, \quad k \geqslant 1 .
$$

These formulas allow one to find the coefficients of the function $f(w)$, whose roots are $c_{i}$. So, the function $f(w)$ is an analog of the resultant for a system of algebraic equations.

\section{$5 \quad$ Examples}

Since the power sums in Theorem 9 are multidimensional series, then, clearly, this theorem provides one with a method for computing multidimensional series of such kind. For this purpose, one needs a system such that the power sums of its roots coincide with terms of the given series. Once such a system has been found, the sum of the series can then be computed by means of Theorem 9 . 
In this section, we consider examples where the described method can be used. In the first example, one can find power sums of roots by applying Theorem 8 . Then, in the second example, we consider infinite products of the functions considered in the first example. Using Theorem 9 we then construct a series consisting of the power sums of the roots of the system and find the sum of this series.

Example 11. Consider the system in two complex variables

$$
\left\{\begin{array}{l}
f_{1}\left(z_{1}, z_{2}\right)=\left(1-a_{2} z_{2}\right)^{2}+a_{3} z_{1} z_{2}^{2}=0 \\
f_{2}\left(z_{1}, z_{2}\right)=\left(1-b_{1} z_{1}\right)^{2}\left(1-b_{2} z_{2}\right)+b_{3} z_{1}^{2} z_{2}=0
\end{array}\right.
$$

with real coefficients $a_{i}$ and $b_{i}$. For this system, $Q_{1}$ and $Q_{2}$ are of the form (10). It is not hard to verify that the system (19) has 5 roots $\left(z_{j 1}, z_{j 2}\right), j=1,2,3,4,5$. If $a_{2} \neq b_{2}$, then all the roots do not lie in the coordinate hyperplanes.

After the substitution $z_{1}=1 / w_{1}, z_{2}=1 / w_{2}$, (19) takes the form

$$
\left\{\begin{array}{l}
\widetilde{f}_{1}=w_{1}\left(w_{2}-a_{2}\right)^{2}+a_{3}=0, \\
\widetilde{f}_{2}=\left(w_{1}-b_{1}\right)^{2}\left(w_{2}-b_{2}\right)+b_{3}=0 .
\end{array}\right.
$$

The Jacobian $\widetilde{\Delta}$ of $(20)$ is equal to

$$
\begin{aligned}
\widetilde{\Delta}=\left|\begin{array}{cc}
\left(w_{2}-a_{2}\right)^{2} & 2 w_{1}\left(w_{2}-a_{2}\right) \\
2\left(w_{1}-b_{1}\right)\left(w_{2}-b_{2}\right) & \left(w_{1}-b_{1}\right)^{2}
\end{array}\right| \\
\quad=\left(w_{1}-b_{1}\right)^{2}\left(w_{2}-a_{2}\right)^{2}-4 w_{1}\left(w_{1}-b_{1}\right)\left(w_{2}-a_{2}\right)\left(w_{2}-b_{2}\right) .
\end{aligned}
$$

Now, using Theorem 8, we compute the power sums

$$
\begin{aligned}
& \sigma_{\gamma}=\sum_{j=1}^{5} \frac{1}{z_{j 1}^{\gamma_{1}+1}} \cdot \frac{1}{z_{j 2}^{\gamma_{2}+1}} \\
& \quad=\sum_{J}(-1)^{s(j)} \sum_{K \in \Re} \frac{(-1)^{\|K\|}}{(2 \pi i)^{2}} \int_{\widetilde{\Gamma}_{h, a_{J}}} \frac{w_{1}^{\gamma_{1}+1} \cdot w_{2}^{\gamma_{2}+1} \cdot a_{3}^{k_{1}} \cdot b_{3}^{k_{2}} \cdot \widetilde{\Delta} \cdot d w_{1} \wedge d w_{2}}{w_{1}^{k_{1}+1}\left(w_{2}-a_{2}\right)^{2\left(k_{1}+1\right)} \cdot\left(w_{1}-b_{1}\right)^{2\left(k_{2}+1\right)}\left(w_{2}-b_{2}\right)^{k_{2}+1}} .
\end{aligned}
$$

Here $\Re=\left\{K=\left(k_{1}, k_{2}\right)\right.$ : there exists $i$ such that $\gamma_{i}+2>k_{1}+k_{2}$ for $\left.i=1,2\right\}$, and $\widetilde{\Gamma}_{h, a_{J}}$ are the cycles either $\left\{\left|w_{1}\right|=r_{11},\left|w_{2}-b_{2}\right|=r_{22}\right\}$ oriented positively or $\left\{\left|w_{2}-a_{2}\right|=r_{12},\left|w_{1}-b_{1}\right|=r_{21}\right\}$ oriented negatively.

In particular, by computing $J_{(0,0)}$, we obtain that

$$
\sigma_{(1,1)}=4 a_{2} b_{1}-\frac{a_{3} b_{2}}{\left(b_{2}-a_{2}\right)^{2}}
$$

without finding the roots.

Example 12. Recall the known expansions of $\sin z$ into an infinite product and a power series:

$$
\frac{\sin \sqrt{z}}{\sqrt{z}}=\prod_{k=1}^{\infty}\left(1-\frac{z}{k^{2} \pi^{2}}\right)=\sum_{k=0}^{\infty} \frac{(-1)^{k} z^{k}}{(2 k+1) !}
$$


Both are absolutely and uniformly convergent on any compact subset of the complex plane.

Consider the system

$$
\left\{\begin{array}{l}
f_{1}=\frac{\sin \sqrt{2 a_{2} z_{2}-a_{2}^{2} z_{2}^{2}-a_{3} z_{1} z_{2}^{2}}}{\sqrt{2 a_{2} z_{2}-a_{2}^{2} z_{2}^{2}-a_{3} z_{1} z_{2}^{2}}}=\prod_{k=1}^{\infty}\left(\left(1-\frac{a_{2} z_{2}}{k^{2} \pi^{2}}\right)^{2}+\frac{a_{3} z_{1} z_{2}^{2}}{k^{2} \pi^{2}}\right)=0 \\
f_{2}=\frac{\sin \sqrt{b_{2} z_{2}+2 b_{1} z_{1}-2 b_{1} b_{2} z_{1} z_{2}-b_{1}^{2} z_{1}^{2}+b_{1}^{2} b_{2} z_{1}^{2} z_{2}-b_{3} z_{1}^{2} z_{2}}}{\sqrt{b_{2} z_{2}+2 b_{1} z_{1}-2 b_{1} b_{2} z_{1} z_{2}-b_{1}^{2} z_{1}^{2}+b_{1}^{2} b_{2} z_{1}^{2} z_{2}-b_{3} z_{1}^{2} z_{2}}} \\
=\prod_{m=1}^{\infty}\left(\left(1-\frac{b_{1} z_{1}}{m^{2} \pi^{2}}\right)^{2}\left(1-\frac{b_{2} z_{2}}{m^{2} \pi^{2}}\right)+\frac{b_{3} z_{1}^{2} z_{2}}{m^{2} \pi^{2}}\right)=0 .
\end{array}\right.
$$

Each function in (22) can be expanded into an infinite product of the functions from (19). The system (22) has infinitely many roots. The assumption $a_{2} \cdot b_{2}<0$ implies that no roots of $(22)$ lie in the coordinate planes.

Using (21), we find that

$$
\sigma_{(1,1)}=\sum_{j=1}^{\infty} \frac{1}{z_{j 1}} \cdot \frac{1}{z_{j 2}}=\sum_{k, m=1}^{\infty} \frac{4 a_{2} b_{1}}{\pi^{4} k^{2} m^{2}}-\sum_{k, m=1}^{\infty} \frac{a_{3} b_{2}}{\pi^{2}\left(a_{2} m^{2}-b_{2} k^{2}\right)^{2}} .
$$

Using the formula [22, No. 2, Section 5.1.25]

$$
\sum_{k=1}^{\infty} \frac{1}{\left(k^{2}+a^{2}\right)^{2}}=\frac{-1}{2 a^{4}}+\frac{\pi}{4 a^{3}} \operatorname{coth}(\pi a)+\frac{\pi^{2}}{4 a^{2}} \cdot \frac{1}{\sinh ^{2}(\pi a)}
$$

we find the sum of the first series in the right hand side of (23)

$$
\sum_{k, m=1}^{\infty} \frac{4 a_{2} b_{1}}{\pi^{4} k^{2} m^{2}}=\frac{a_{2} b_{1}}{9}
$$

and, respectively, the sum of the second series in the right hand side of (23)

$$
\begin{aligned}
\sum_{k, m=1}^{\infty} \frac{a_{3} b_{2}}{\pi^{2}\left(a_{2} m^{2}-b_{2} k^{2}\right)^{2}} & =-\sum_{k=1}^{\infty} \frac{a_{3}}{2 \pi^{2} b_{2} k^{4}} \\
-\sum_{k=1}^{\infty} & \frac{a_{3}}{4 \pi \sqrt{-a_{2} b_{2}} k^{3}} \operatorname{coth}\left(\pi \sqrt{-b_{2} / a_{2}} k\right)-\sum_{k=1}^{\infty} \frac{a_{3}}{4 a_{2} k^{2}} \cdot \frac{1}{\sinh ^{2}\left(\pi \sqrt{\left.-b_{2} / a_{2} k\right)}\right.} .
\end{aligned}
$$

Here the sum of the first series in the right hand side is

$$
\sum_{k=1}^{\infty} \frac{a_{3}}{2 \pi^{2} b_{2} k^{4}}=\frac{a_{3} \pi^{2}}{180 b_{2}}
$$

We now compute the sum of the second series. Let ${ }_{2} \Phi_{1}\left(e^{2 t}, e^{2 t} ; e^{4 t}, x\right)$ be a basic hypergeometric series (see, e.g., [22, p. 793]). We use the known formula [22, No. 13, Section 5.2.18]

$$
\begin{aligned}
\sum_{k=1}^{\infty} \frac{x^{k-1}}{e^{2 t k}-1}=\frac{1}{x} \sum_{k=1}^{\infty} \frac{x^{k}}{e^{2 t k}-1} & \\
= & \frac{1}{x} \cdot \frac{x}{e^{4 t}-1}{ }_{2} \Phi_{1}\left(e^{2 t}, e^{2 t} ; e^{4 t}, x\right)=\frac{1}{e^{4 t}-1}{ }_{2} \Phi_{1}\left(e^{2 t}, e^{2 t} ; e^{4 t}, x\right)
\end{aligned}
$$


Therefore,

$$
\begin{aligned}
& \sum_{k=1}^{\infty} \frac{\operatorname{coth}(t k)}{k^{3}}= \sum_{k=1}^{\infty} \frac{1}{k^{3}}+2 \sum_{k=1}^{\infty} \frac{1}{k^{3}\left(e^{2 t s}-1\right)} \\
&=\zeta(3)+2 \frac{1}{e^{4 t}-1} \int_{0}^{1} \frac{1}{y} d y \int_{0}^{y} \frac{1}{v} d v \int_{0}^{v}{ }_{2} \Phi_{1}\left(e^{2 t}, e^{2 t} ; e^{4 t}, u\right) d u \\
&=\zeta(3)+\frac{1}{e^{4 t}-1} \int_{0}^{1} \ln ^{2} y \cdot{ }_{2} \Phi_{1}\left(e^{2 t}, e^{2 t} ; e^{4 t}, y\right) d y
\end{aligned}
$$

In order to find the sum of the third series we rewrite $\frac{1}{\sinh ^{2}(t k)}$ as

$$
\frac{1}{\sin h^{2}(t k)}=\left(\frac{2}{e^{t k}-e^{-t k}}\right)^{2}=\frac{4 e^{2 t k}}{\left(e^{2 t k}-1\right)^{2}}
$$

Now, since

$$
\frac{\partial}{\partial t}\left[\frac{1}{e^{t k}-1}\right]=-\frac{2 k e^{2 t k}}{\left(e^{2 t k}-1\right)^{2}},
$$

we have

$$
\frac{1}{\left(e^{2 t k}-1\right)^{2}}=-\frac{1}{e^{2 t k}-1}-\frac{1}{2 k} \cdot \frac{\partial}{\partial t}\left[\frac{1}{e^{2 t k}-1}\right] \text {. }
$$

Consequently,

$$
\sum_{k=1}^{\infty} \frac{1}{\sinh ^{2}(t k) \cdot k^{2}}=-\frac{\partial}{\partial t}\left[\sum_{k=1}^{\infty} \frac{2}{k^{3}\left(e^{2 t k}-1\right)}\right]
$$

Thus, using the formula (24), we express the series $\sigma_{(1,1)}$ in terms of the values of some integrals and known series without calculating the roots of the system.

\section{Disclosure statement}

No potential conflict of interest was reported by the authors.

\section{Funding}

This research was supported by grants of the President of the Russian Federation for young scientists, project MD-197.2017.1 and for leading scientific schools, project NSh-9149.2016.1, by grant of the Government of the Russian Federation for investigations under the guidance of the leading scientists of the Siberian Federal University (contract No. 14.Y26.31.0006), and by the Russian Foundation for Basic Research, project 18-01-00024-a.

\section{References}

[1] Adams W. W., Loustaunau P. An introduction to Gröbner bases. Graduate Studies in Mathematics. Vol. 3. Providence (RI): Amer. Math. Soc.; 1994. 
[2] Aizenberg L. A. On a formula of the gereralized multidimensional logarithmic residue and the solution of system of nonlinear equations. Sov. Math. Doc. 1977;18:691-695.

[3] Aizenberg L. A., Yuzhakov A. P. Integral representations and residues in multidimensional complex analysis. Translations of Mathematical Monographs. Providence (RI): Amer. Math. Soc.; 1983.

[4] Buchberger B. Gröbner bases, an algorithmic method in polynomial ideal theory. In Multidimensional Systems Theory (N. K. Bose ed.). Reidel, Dordrecht; 1985. p. 184-232.

[5] Bykov V., Kytmanov A., Lazman M., and Passare M. (ed). Elimination Methods in Polynomial Computer Algebra. Math. and Appl., Vol. 448, Kluwer Acad. Publ. Dordreht, Boston, London; 1998.

[6] Bykov V. I. Modeling of the critical phenomena in chemical kinetics. Moscow: Komkniga (in Russian); 2006.

[7] Bykov V. I., Kytmanov A. M., Myslivets S. G. Power sums of nonlinear systems of equations. Docl. Math. 2007;76(2):641-645.

[8] Bykov V. I, Tsybenova S. B. Non-linear models of chemical kinetics. Moscow: Krasand (in Russian); 2011.

[9] Khodos O. V. On some systems of non-algebraic equations in $\mathbb{C}^{n}$. Journal of Siberian Federal University - Mathematics and Physics 2014;7(4):455-465.

[10] Kulikov V. R., Stepanenko V. A. On solutions and Waring's formulas for systems of $n$ algebraic equations for $n$ unknowns. St. Petersburg Math. J. 2015;26(5):839-848.

[11] Kytmanov A. A. Analogs of Recurrent Newton Formulas. Russian Mathematics 2009;53(10):34-44.

[12] Kytmanov A. A. An Algorithm for Calculating Power Sums of Roots for a Class of Systems of Nonlinear Equations. Program. Comput. Soft. 2010;36(2):103-110.

[13] Kytmanov A. A., Kytmanov A. M., Myshkina E. K. Finding Residue Integrals for Systems of Non-algebraic Equations in $\mathbb{C}^{n}$. Journal of Symbolic Computation 2015;66:98-110.

[14] Kytmanov A. M., Khodos O. V. On systems of non-algebraic equation in $\mathbb{C}^{n}$. 1st USAUzbekistan Conference on Analysis and Mathematical Physics, in Contemporary Math. 2016;662:77-88.

[15] Kytmanov A. M, Myshkina E. K. Evaluation of power sums of roots for systems of nonalgebraic equations in $\mathbb{C}^{n}$. Russian Mathematics 2013;57(12):31-43.

[16] Kytmanov A. M, Myshkina E. K. On calculation of power sums of roots one class of systems non-algebraic equations. Siberian Electronic Mathematical Reports (in Russian) 2015;12:190-209.

[17] Kytmanov A. M, Myshkina E. K. Power series of roots of systems of entire functions of finite growth order. Journal of Mathematical Sciences (United States) 2016;213(6):868-886. 
[18] Kytmanov A. M, Potapova Z. E. Formulas for determining power sums of roots of systems of meromorphic functions. Izvestiya VUZ. Matematika (in Russian) 2005;49(8):36-45.

[19] Lelong P., Gruman L. Entire Functions of Several Complex Variables. Berlin, Heidelberg, New York: Springer-Verlag; 1986.

[20] Myshkina E. K. On One Condition for the Decomposition of an Entire Function into an Infinite Product. Journal of Siberian Federal University - Mathematics and Physics 2014;7(1):91-94.

[21] Passare M., Tsikh A. Residue integrals and their Melin transforms. Can. J. Math. 1995;47(5):1037-1050.

[22] Prudnikov A. P., Brychkov Yu. A., Marichev O. I. Integrals and series. Vol. 1. Elementary functions. Translated from the Russian and with a preface by Queen NM. New York: Gordon \& Breach Science Publishers; 1986.

[23] Tsikh A. K. The Bezout theorem in the space of theory functions. On the solution of a system of algebraic equations. Nekotorye voprosy mnogomernogo kompleksnogo analiza (in Russian). Krasnoyarsk: Institut fiziki SO AN SSSR; 1980. p. 185-196.

[24] Tsikh A. K. Multidimensional residues and their applications. Translations of Mathematical Monographs. Vol. 103. Providence (RI): Amer. Math. Soc.; 1992.

[25] Van der Waerden B. L. Modern algebra. New York: Frederick Unger Publishing Co.; 1950. 